\title{
El éxito del mantenimiento productivo total y su relación con los factores administrativos
}

\author{
Jorge Luis García \\ Alcaraz \\ Instituto de Ingeniería y \\ Tecnología, Universidad Autó- \\ noma de Ciudad Juárez \\ jorge.garcia@uacj.mx \\ Jaime Romero González \\ Instituto de Ingeniería y \\ Tecnología, Universidad Autó- \\ noma de Ciudad Juárez \\ jromero@uacj.mx

\section{Salvador Anacleto} \\ Noriega Morales \\ Instituto de Ingeniería y \\ Tecnología, Universidad Autó- \\ noma de Ciudad Juárez \\ snoriega@uacj.mx
}

\section{Resumen}

El mantenimiento productivo total es una herramienta ampliamente usada en las áreas productivas, la cual está encaminada a incrementar la disponibilidad de la maquinaria y equipo de producción, así como los beneficios económicos de las empresas. Sin embargo, no se conocen los factores administrativos que aseguren su éxito de implantación. En este artículo se presentan los resultados de una encuesta — que fue aplicada a 203 gerentes y supervisores de mantenimiento de empresas localizadas en Ciudad Juárez- que constó de veinte actividades y tres beneficios, y que debía ser respondida en una escala Likert. El cuestionario se validó mediante el índice alfa de Cronbach y se aplicó un análisis factorial exploratorio mediante el método de componentes principales y se determinó que solamente tres factores podían explicar el $66.06 \%$ de la varianza total, mientras que la relación entre los factores, las actividades y beneficios se obtuvieron mediante modelos de ecuaciones estructurales.

Palabras clave: TPM, factores clave del éxito, análisis factorial, modelo de ecuaciones estructurales. 


\title{
Administrative factors related to the success of total productive maintenance
}

\begin{abstract}
Total Productive Maintenance is a tool widely used in the production areas. It is aimed at increasing the availability of machinery and production equipment, as well as businesses economic benefits. However, the administrative factors that ensure its successful implementation are not known. This article presents the results of a survey with twenty activities and three benefits. This survey was applied to 203 managers and maintenance supervisors from companies located in Ciudad Juarez, and was to be answered on a Likert scale. The survey was validated by the use of Cronbach's alpha index and an exploratory principal components method factorial analysis was applied. We found that only 3 factors can explain $66.06 \%$ of the total variance, while the relationship between the factors, activities and benefits was obtained with the use of structural equation models.
\end{abstract}

Keywords: TPM, critical success factors, factor analysis, structural equation models.

\section{Introducción}

El Mantenimiento Preventivo Total (TPM, Total Productive Maintenance, por sus siglas en inglés) es en la actualidad una de las principales herramientas para lograr la eficiencia y competitividad, lo que supone cumplir con especificaciones de calidad, tiempo y costo de la producción; generalmente se ejecuta junto con la Administración Total de la Calidad (TQM, Total Quality Management, por sus siglas en inglés), que se fundamenta en la búsqueda permanente por mejorar los rendimientos de procesos y los medios de producción (Wikoff, 2007). Además, el TPM es altamente eficaz en aquellas empresas que cuentan con operaciones automáticas y secuenciales (empresas intensivas en el uso de maquinaria), y no requiere grandes inversiones económicas para lograr el aprovechamiento de las instalaciones existentes (Cooke, 2000; Wikoff, 2007).

El TPM no es una idea nueva, es simplemente el siguiente paso en la evolución de las buenas prácticas de mantenimiento (Dinesh y Triphati, 2006; Wikoff, 2007). En algunas ocasiones se confunden las diferencias entre el mantenimiento predictivo PM (preventivo, predictivo y proactivo) y TPM, pero este último hace uso del ciclo de vida del producto y del equipo, lo que reduce al mínimo el número de fallas del mismo, los defectos de producción y los accidentes; el TPM se define como una estrategia agresiva que se centra en mejorar la función y el diseño de la 
producción (Chandra y Shastri, 1998; Wikoff, 2007) e involucra a cada persona en la organización.

Así, se concluye que el TPM pretende aumentar la disponibilidad y eficacia de la maquinaria y equipo manteniéndolo en el nivel óptimo de servicio e incrementar su ciclo de vida; por ende, también con la inversión mínima en recurso humanos (Cooke, 2000), lo que permite disminuir y controlar la variación en el proceso de producción (Reed, 1996).

Cuando no se implementa adecuadamente el TPM, entonces se corre el riesgo de incurrir en las seis grandes pérdidas, las cuales se manifiestan en tres aspectos fundamentales (Tajiri y Gotoh, 1992): tiempos muertos o paro del sistema productivo, funcionamiento a velocidad inferior a la capacidad de los equipos y productos defectuosos o malfuncionamiento de las operaciones en un equipo. Las seis grandes pérdidas son las averías frecuentes de la maquinaria, grandes tiempos en preparaciones entre un lote y otro, microparadas (las cuales acumuladas suman bastante tiempo), velocidad de proceso menor y frecuentes obstáculos, calidad reducida del producto final y tiempos elevados de arranque.

Sin embargo, cuando el TPM se implanta adecuadamente, éste tiene numerosos beneficios para la empresa, entre los que se pueden mencionar los siguientes:

- Los gastos de mantenimiento son planeados y controlados (Tajiri y Gotoh, 1992; Eti et al., 2004), lo que permite reducir la mano de obra en mantenimiento.

- Reducción de la mano de obra indirecta (Takahashi y Osada, 1989; Chan et al., 2005).

- Mejoran las relaciones entre los operadores, los cuales son reconocidos por las ideas que aportan a la empresa (Chand y Shirvani, 2000; Cua et al., 2001; Gosavi, 2001; McKone et al., 2001; Chan et al., 2005; Aichlmayr, 2009).

- Se incrementa la calidad del producto y la satisfacción del cliente (Ashayeri, 2007).

- Dado que el equipo es más confiable y el proceso más repetible, la programación del flujo de trabajo se vuelve más fácil (Alsyouf, 2009; Dowlatshahi, 2009). 
De la misma manera, se encuentran varios casos de estudio en que se reportan los resultados de aplicar TPM en las empresas: McKone et al. (1999) analizan las organizaciones americanas que lo han implantado y relatan sus beneficios; Eti et al. (2004) señalan el proceso de implantación en una planta industrial de Nigeria y los beneficios que han alcanzado; Sachdeva et al. (2008) muestran cómo se han optimizado secciones de una empresa dedicada a la producción de papel; por su parte, Dowlatshahi (2008) realizó un análisis empírico del rol que juega el TPM en la industria maquiladora; Pinjala et al. (2006) analizaron la importancia del TPM y han concluido que éste debe ser parte de la estrategia de muchas empresas si desean ser competitivas; Vinodh (2010) reporta un caso de estudio donde indica cómo se puede lograr un sistema de producción ágil y sustentable mediante la aplicación de TPM, lo cual ya había sido mencionado anteriormente por Cua et al. (2001) al relacionar los beneficios del TPM con justo a tiempo (JIT, just in time, por sus siglas en inglés) y Control Total de la Calidad.

Sin lugar a dudas, son muchos los beneficios que ofrece el TPM cuando éste es bien implantado en las empresas; sin embargo, la pregunta de todo gerente de mantenimiento es ¿qué debe hacerse para alcanzar tales beneficios reportados en la literatura?, específicamente ¿qué debe hacer la gerencia y sus empleados para alcanzar dichos beneficios? Lamentablemente muchos autores se han limitado a definir procesos de implantación y generalmente son estudios de caso particulares, por ejemplo, “cómo he implementado el TPM en mi compañía?” (Eti et al., 2006; Pinjala et al., 2006; Zhou y Zhu, 2008; Aissani et al., 2009); dichos autores fueron generalmente los empleados (o consultores) responsables de la implantación del TPM en la empresa. Desafortunadamente, la mayoría de éstos son casos aislados de observaciones o sucesos en un determinado lugar y tiempo, y pocas investigaciones se han realizado en relación con los factores clave del éxito de la herramienta, específicamente aquellos de tipo administrativo.

Por ello, el objetivo de esta investigación es presentar un análisis descriptivo y multivariado en el que se identifiquen los factores administrativos claves del éxito del TPM y determinar las relaciones que éstos tienen, lo cual ayudará a las empresas a realizar una mejor planeación del proceso de adopción de esta técnica y lograr sus beneficios. 


\section{Metodología}

La metodología empleada en esta investigación ha implicado el diseño de un instrumento de recolección de datos, así como la identificación de actividades de tipo administrativo que son consideradas importantes en el éxito del TPM. Asimismo, se recolectó y analizó la información para llegar a una conclusión con base en los resultados encontrados; así, se ha trabajado en diferentes etapas que se describen a continuación.

\section{Primera etapa. Identificación de las actividades y creación del instrumento}

Esta etapa se focalizó en una revisión bibliográfica para identificar las investigaciones relacionadas con el problema y determinar las actividades administrativas que se deben realizar para garantizar el éxito del TPM; se encontró referencia a diecisiete de éstas y dos principales beneficios con los cuales se elaboró un cuestionario preliminar que fue aplicado a un total de ochenta y ocho gerentes o supervisores de TPM de empresas localizadas en Ciudad Juárez, Chihuahua. Sin embargo, se dejó espacio para que los encuestados manifestaran otras actividades requeridas y beneficios que a su juicio eran importantes y que no aparecían en el cuestionario inicial. Un total de tres nuevas actividades y un beneficio fueron identificados; de esta forma se obtuvieron un total de veinte actividades y tres beneficios, lo que permitió construir un cuestionario final.

El cuestionario final debía ser contestado en una escala Likert (Likert, 1932), que comprendía valores entre uno y nueve, donde el uno indicaba la falta de importancia y el nueve la importancia extrema. Las actividades y beneficios se ilustran en el cuadro 1 con una abreviatura que es utilizada a lo largo del trabajo. 


\section{Cuadro 1}

\section{Actividades y sus abreviaciones}

\begin{tabular}{|c|c|}
\hline Abreviación & Actividad \\
\hline HerraLugar & Poner todas las herramientas y accesorios en su lugar \\
\hline TjoLimOrde & Mantener áreas de trabajos limpios y ordenados \\
\hline EmpDtoLimp & Empresa limpia en todo momento y en todos los departamentos \\
\hline EntrMultTar & Entrenamiento para que empleados realicen adecuadamente múltiples tareas \\
\hline EmplRespMtto & Responsabilidad de empleados en el mantenimiento de las máquinas que operan \\
\hline EmplSuplir & Entrenamiento de empleados para que puedan suplir a otros si es necesario \\
\hline EmpIdeaMantto & $\begin{array}{l}\text { Los operadores proporcionen opiniones e ideas antes de tomar una decisión } \\
\text { relacionada con mantenimiento }\end{array}$ \\
\hline BitaMaq & Llevar una bitácora de trabajo y mantenimiento de cada máquina \\
\hline ConcenMatto & $\begin{array}{l}\text { Programación de mantenimientos en consenso con el departamento de } \\
\text { producción }\end{array}$ \\
\hline ProgMtoOpera & Programación del mantenimiento de máquinas con opinión de los operadores \\
\hline OperOpiBita & Cooperación del operador en redacción de los reportes en bitácora \\
\hline OperSolMto & El operador solicita mantenimiento directamente al departamento \\
\hline OperStopProd & El operador para una línea de producción si detecta problemas de mantenimiento \\
\hline GerenEjem & La alta gerencia es ejemplo del orden y limpieza en el área de trabajo \\
\hline JefesRespMto & Los jefes de departamento aceptan su responsabilidad hacia el TPM \\
\hline LiderMto & Liderazgo de la alta gerencia en la ejecución de los programas de TPM \\
\hline ReuProdMto & Reuniones de trabajo entre el departamento de mantenimiento y producción \\
\hline DirPromParTrab & $\begin{array}{l}\text { Promoción por la gerencia de la participación de trabajadores en mantenimiento } \\
\text { y conservación del equipo }\end{array}$ \\
\hline VisCalYMto & $\begin{array}{l}\text { Creación y comunicación de la dirección de una visión centrada en calidad y } \\
\text { mantenimiento }\end{array}$ \\
\hline \multirow[t]{2}{*}{ DirInvolMto } & Involucramiento de la dirección en proyectos de mantenimiento \\
\hline & Beneficio \\
\hline MaqComp & La maquinaria y equipo de la empresa ayudan a obtener una ventaja competitiva \\
\hline MattoEstra & $\begin{array}{l}\text { El mantenimiento es una estrategia para la calidad y la programación de } \\
\text { actividades }\end{array}$ \\
\hline AcptTPMRes & Todo el personal dentro de la empresa acepta su responsabilidad hacia el TPM \\
\hline
\end{tabular}

\section{Segunda etapa. Aplicación del cuestionario}

Esta etapa consistió en contactar vía telefónica a los candidatos que responderían el cuestionario, los cuales laboraban en empresas del corredor industrial de Ciudad Juárez, Chihuahua, México. Para ello se usó un directorio de empresas y personal proporcionado por la AMAC (Asociación de Maquiladoras, A. C.) que constaba de 769 gerentes y supervisores de mantenimiento. Se acordó una cita con todos los posibles encuestados para realizar el llenado del cuestionario y se acudió al lugar de trabajo de éstos; sin embargo, algunos de ellos prefirieron que se les enviara vía correo electrónico. 
Tercera etapa. Captura de la información y validación del instrumento

En esta etapa se capturó y analizó la información en el software denominado SPSS 18. Para la validación del cuestionario se usó el índice alfa de Cronbach antes de realizar un análisis y se validó el resultado obtenido comparándolo con el generado al realizar la partición de la muestra en mitades, obteniéndose nuevamente el índice alfa de Cronbach (Cronbach, 1951).

\section{Cuarta etapa. Análisis de la información}

Esta etapa se focalizó en realizar el análisis de la información. Se obtuvo la mediana y la moda como medidas de tendencia central, dado que los datos obtenidos, aunque eran numéricos, estaban representados en una escala ordinal (Denneberg y Grabisch, 2004; Pollandt y Wille, 2005; Tastle y Wierman, 2007). Altos valores de la mediana indican que esas actividades tienen mucha importancia en el éxito de TPM y, por el contrario, valores bajos denotaban poca importancia. En relación con la moda, los valores obtenidos en las actividades indican el consenso o valor que más fue respondido por los encuestados.

De la misma manera, como medida de posición se estimaron el primer y tercer cuartil, así como la diferencia entre éstos, el cual se denomina rango intercuartílico (RI) y representa el 50\% de los datos e incluye a la mediana, el cual está representado por el segundo cuartil (Tastle y Wierman, 2007). Altos valores en el rango intercuartílico indican que no había consenso entre los encuestados en relación con el nivel de importancia que tenía esa actividad, mientras que valores bajos representaban poca dispersión de los datos y, por ende, mayor consenso entre los encuestados con relación a la importancia de la actividad.

\section{Quinta etapa: Análisis factorial exploratorio (AFE)}

En esta etapa se determinó la factibilidad del AFE, se analizó la matriz de correlaciones y se observó que la mayoría de las correlaciones entre las actividades eran mayores a 0.3 (Nunally, 1978; Nunnally y Bernstein, 2005); además, se analizó la diagonal de la matriz antimagen de la matriz de correlaciones con la finalidad de observar la adecuación de la muestra. Asimismo, se obtuvo el índice KMO (Kaiser-Meyer-Olkin), se aplicó la prueba de esfericidad de Bartlett para medir la adecuación de la muestra y se analizaron las comunalidades de cada una de las 
actividades para analizar su contribución, estableciendo 0.5 como punto de corte (Lévy y Varela, 2003).

Para determinar los factores críticos o variables latentes, se realizó un análisis factorial por el método de componentes principales usando la matriz de correlación para la extracción de los componentes y se consideraron como importantes aquellos factores con un valor mayor o igual a la unidad en sus eigenvalores, condicionándose la búsqueda a 100 iteraciones para la convergencia de un resultado (Streiner y Norman, 1995). Además, con la finalidad de obtener una mejor interpretación de los factores críticos, se realizó una rotación por el método Varimax (Lévy y Varela, 2003). Las actividades que integran los factores se identificaron mediante los altos valores que contenían en las cargas factoriales, la cual es una medida de correlación con el eje factorial (Nunnally y Bernstein, 2005).

\section{Sexta etapa. Modelo de ecuaciones estructurales}

Con la finalidad de determinar las relaciones entre los factores encontrados en el AFE y analizar el impacto que tenían sobre los tres beneficios identificados, se generó un modelo de ecuaciones estructurales (MEE), para lo cual se usó el software denominado AMOS 18. Las variables que ingresaron al modelo fueron aquellas que contenían una carga factorial mayor a 0.55 en el análisis factorial (Marsh y Hocevar, 1985; Byrne, 1989).

En los MEE, los óvalos representan variables latentes o no observadas, mientras que los rectángulos son las actividades que fueron observadas o medidas; de la misma manera, se usan flechas en un solo sentido, para indicar la dependencia de una variable con otra, y las de doble sentido, en las que no se puede definir una dependencia entre las variables. Así, por ejemplo, si una flecha "sale" de una variable uno con dirección a una variable dos, ello indica que la variable dos depende de la variable uno, la cual es independiente, por lo que se puede decir que una variable es independiente de todas las demás si no existen relaciones mediante flechas que "entren a ella".

Para validar las relaciones entre factores y actividades se analizaron los valores obtenidos para cada uno de los factores y el error estándar de los mismos; además, se obtuvo una relación crítica de cada estimación, haciendo inferencias al 95\% de confianza sobre los valores de los parámetros para determinar si éstos eran triviales 
o no; para ello, se basó en el teorema del límite central (Jöreskog y Sörbom, 1984; Tanaka y Huba, 1985), lo que permitió eliminar relaciones triviales.

Asimismo, con la finalidad de medir la eficiencia de los MEE, se usó el mínimo valor de la chi cuadrada ( $\chi^{2}$, CMIN), los grados de libertad del modelo (DF) y la relación de estos dos parámetros (CMIN/DF) (Wheaton et al., 1977; Carmines y McIver, 1981; Marsh y Hocevar, 1985; Byrne, 1989). De igual forma, con la finalidad de tener un modelo suficientemente explicativo, se usó el índice de bondad de ajuste (GFI), que es una medida de eficiencia y se recomienda que tenga valores superiores a 0.9 (Jöreskog y Sörbom, 1984; Tanaka y Huba, 1985).

El mejor modelo se generó de manera iterativa en función de los índices de modificación, añadiendo relaciones que ayudaban a reducir el valor de la $\chi^{2}$ (error del modelo) y con la pérdida mínima de grados de libertad (Jöreskog y Sörbom, 1989). Asimismo, se observó la mejoría en la probabilidad del error tipo I (p), el cual fue diferente en cada modelo (Cochran, 1952; Gulliksen y Tukey, 1958; Jöreskog, 1969). También se analizó el índice comparativo de ajuste (CFI) con la finalidad de analizar la mejoría entre un modelo y otro, aceptando un modelo sobre otro si la diferencia del CFI es mayor a 0.01 (Bentler, 1990; McDonald y Marsh, 1990) y se buscó mantener una medida aceptable de la raíz cuadrada media residual (RMSEA), que debe ser menor a 0.05 (Steiger y Lind, 1985; Browne y Cudeck, 1993).

Con la finalidad de observar la adecuación del tamaño de la muestra para el modelo y asegurar que las modificaciones eran válidas, y no se violara la restricción del tamaño de muestra que se había logrado obtener, se analizó el índice crítico $\mathrm{N}$ de Hoelter con un nivel de confianza del 95\% (Hoelter, 1983; Bollen y Liang, 1988).

\section{Resultados}

La sección de resultados está dividida en subsecciones, según la información que se presente.

\section{Composición de la muestra}

En el periodo de recolección de información se obtuvo un total de 203 cuestionarios válidos; de éstos 72 provenían de empresas ubicadas en los corredores industriales de Ciudad Juárez, Chihuahua, México, de los que 68 pertenecían a gerentes 
o superintendentes de mantenimiento; el resto, 135, provenían de supervisores de la misma área. En la figura 1 se ilustra el tipo de empresa o sector al que estaban adscritos los 203 encuestados, donde claramente se observa que el sector automotriz y eléctrico/electrónica son los más representados.

\section{Figura 1}

Sectores encuestados

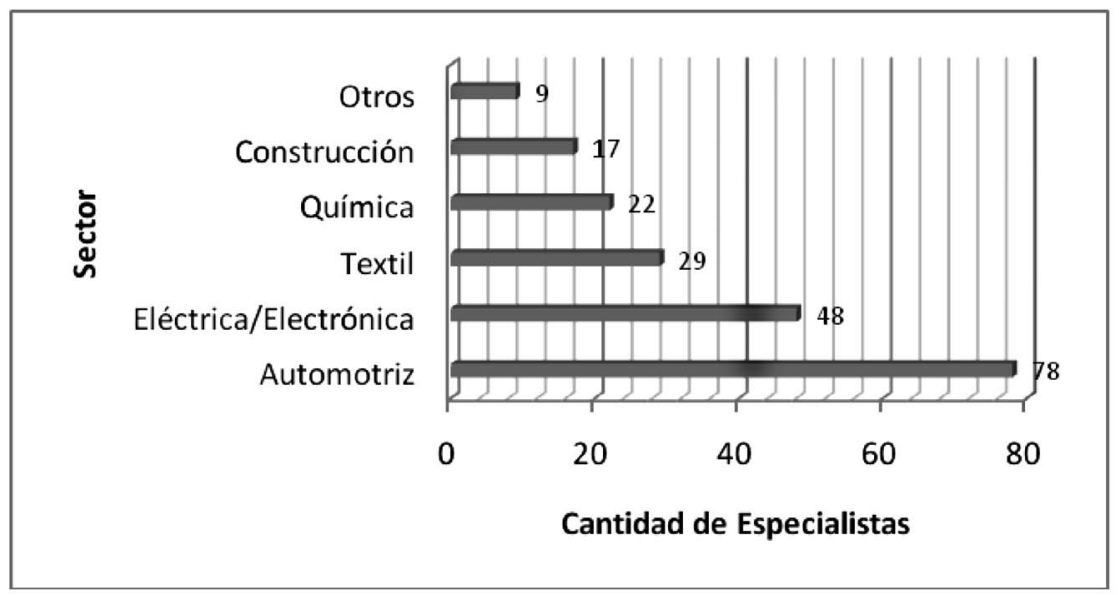

De la misma manera, en la figura 2 se exponen las profesiones que tenían los encuestados responsables de las actividades de mantenimiento. Se observa claramente que la carrera de ingeniería industrial es la más representada, seguida por ingeniería mecánica, ingeniería electrónica y mecatrónica.

Validación del cuestionario, construcción de escala e identificación de actividades

Se obtuvo el índice alfa de Cronbach en las veinte actividades iniciales y se observó que eliminando algunas de éstas se mantenía o mejoraba la consistencia interna del cuestionario, por lo que la lista final de actividades por analizar se obtuvo de manera iterativa; es decir, se eliminaban actividades del cuestionario si éste mejoraba su consistencia interna. En el cuadro 2 se listan las actividades en la primera columna; después, se ilustran las diferentes iteraciones y el valor del índice alfa de Cronbach si esa actividad se eliminaba, las cuales se indican con un asterisco (*). 
Figura 2

Profesiones de los encuestados

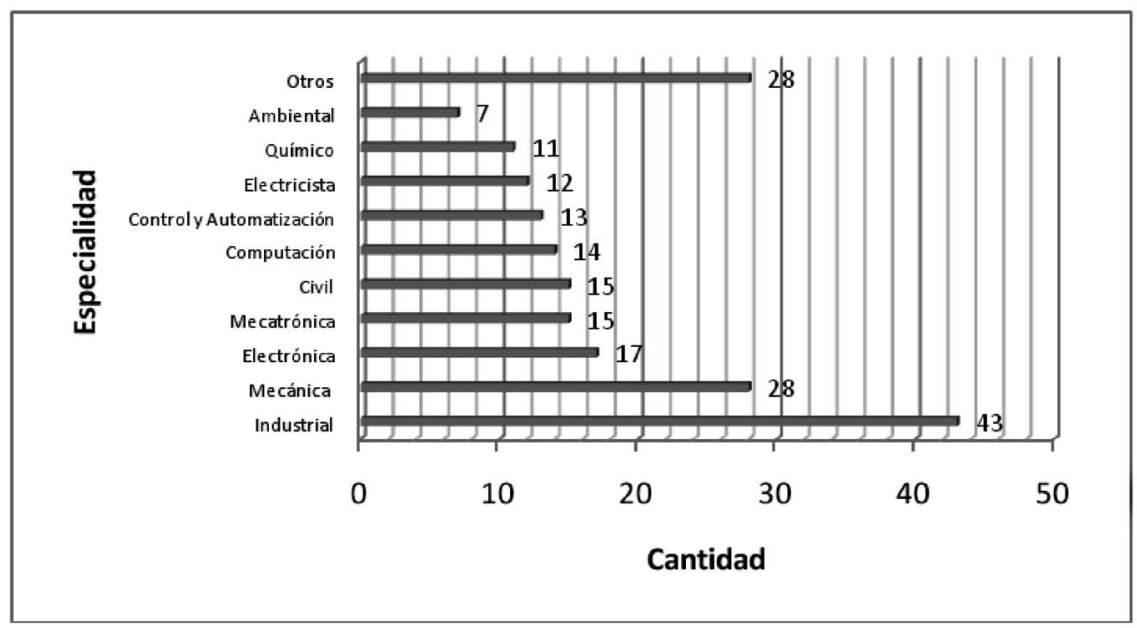

Cuadro 2

Validación del instrumento y construcción de escala

\begin{tabular}{llllllll}
\hline \multicolumn{1}{l}{ Ítem } & & Alfa & \multicolumn{3}{c}{ Cronbach si el ítem es eliminado (Iteración) } \\
\hline 1. & HerraLugar & 0.912 & 0.912 & 0.911 & 0.911 & 0.912 & 0.912 \\
2. & TjoLimOrde & 0.912 & 0.912 & 0.912 & 0.911 & 0.912 & 0.912 \\
3. & EmpDtoLimp & 0.911 & 0.911 & 0.911 & 0.91 & 0.911 & 0.911 \\
4. & EntrMultTar & 0.91 & 0.909 & 0.909 & 0.908 & 0.909 & 0.909 \\
5. & EmplSuplir & 0.911 & 0.911 & 0.911 & 0.911 & 0.912 & 0.912 \\
6. & ProgMtoOpera & 0.912 & 0.912 & 0.911 & 0.911 & 0.912 & 0.913 \\
7. & OperOpiBita & 0.913 & 0.913 & 0.912 & 0.912 & 0.914 & 0.914 \\
8. & OperSolMto & 0.912 & 0.912 & 0.912 & 0.911 & 0.912 & 0.913 \\
9. & GerenEjem & 0.913 & 0.913 & 0.912 & 0.912 & 0.913 & 0.913 \\
10. & JefesRespMto & 0.908 & 0.907 & 0.906 & 0.905 & 0.906 & 0.906 \\
11. & LiderMto & 0.906 & 0.906 & 0.905 & 0.904 & 0.904 & 0.903 \\
12. & ReuProdMto & 0.908 & 0.908 & 0.907 & 0.906 & 0.907 & 0.907 \\
13. & DirPromParTrab & 0.908 & 0.907 & 0.906 & 0.905 & 0.905 & 0.905 \\
14. & VisCalYMto & 0.908 & 0.907 & 0.906 & 0.905 & 0.905 & 0.905 \\
15. & DirInvolMto & 0.909 & 0.908 & 0.908 & 0.907 & 0.907 & 0.907 \\
16. & EmplRespMtto & 0.914 & 0.914 & 0.914 & $0.915 *$ & & \\
17. & EmpIdeaMantto & 0.915 & 0.915 & $0.915 *$ & & & \\
18. & BitaMaq & $0.915 *$ & & & & & \\
19. & ConcenMatto & 0.914 & 0.915 & 0.915 & 0.914 & $0.915 *$ & \\
20. & OperStopProd & 0.915 & $0.915 *$ & & & & \\
\hline Alfa de Cronbach Total & 0.915 & 0.915 & 0.915 & 0.915 & 0.915 & 0.915 \\
\hline
\end{tabular}


En el cuadro 2 se observa que el índice alfa de Cronbach (IAC) alcanzó un valor grupal (con las veinte actividades) de 0.915 en la primera iteración y que eliminando la actividad denotada por BitaMaq se obtiene el mismo valor (0.915), por lo que se eliminó del análisis y se volvió a obtener el IAC en la segunda iteración. Ahora, se observa que eliminando la actividad OperStopProd no se altera el valor del índice grupal, ya que se obtiene el mismo valor de 0.915 , por lo que se eliminó y se procedió con la tercera iteración. Después de seis iteraciones, se observa que se han eliminado cinco actividades del análisis y que no se mejora el IAC grupal al eliminar otra, por lo que el proceso iterativo termina y los análisis sucesivos sólo se realizan en las quince actividades restantes.

\section{Análisis de la muestra}

En el cuadro 3 aparecen las quince actividades que forman la escala de medición de los aspectos administrativos de TPM y se exponen la mediana, la moda, el percentil 25, el percentil 75 y el rango intercuartílico (RI). De este análisis univariable se puede apreciar que las actividades que alcanzaron calificaciones más altas en relación con la mediana fueron poner todas las herramientas y accesorios en su lugar, que la alta gerencia sea ejemplo de orden y limpieza, que ésta promueva la participación de trabajadores en mantenimiento y conservación de equipo, y que se mantenga un área de trabajo limpio y ordenado.

\section{Cuadro 3}

Análisis descriptivo de la muestra

\begin{tabular}{|c|c|c|c|c|c|}
\hline \multirow{2}{*}{ Actividad } & \multirow{2}{*}{ Mediana } & \multirow{2}{*}{ Moda } & \multicolumn{2}{|c|}{ Percentiles } & \multirow{2}{*}{ RI } \\
\hline & & & 25 & 75 & \\
\hline HerraLugar & 7.53 & 9 & 4.86 & 8.86 & 4.01 \\
\hline GerenEjem & 7.04 & 9 & 4.71 & 8.48 & 3.77 \\
\hline DirPromParTrab & 6.75 & 9 & 4.15 & 8.35 & 4.21 \\
\hline TjoLimOrde & 6.73 & 7 & 4.80 & 8.15 & $3.35 *$ \\
\hline VisCalYMto & 6.65 & 7 & 4.40 & 8.15 & 3.75 \\
\hline EntrMultTar & 6.60 & 9 & 4.70 & 8.41 & $3.72 *$ \\
\hline EmpDtoLimp & 6.54 & 5 & 4.87 & 8.29 & $3.42 *$ \\
\hline LiderMto & 6.51 & 7 & 3.44 & 8.15 & 4.71 \\
\hline OperSolMto & 6.50 & 9 & 3.22 & 8.49 & 5.27 \\
\hline EmplSuplir & 6.48 & 9 & 4.39 & 8.28 & 3.89 \\
\hline JefesRespMto & 6.34 & 9 & 3.40 & 8.24 & 4.83 \\
\hline ReuProdMto & 6.24 & 9 & 3.11 & 8.12 & 5.01 \\
\hline DirInvolMto & 5.46 & 7 & 2.61 & 7.60 & 4.99 \\
\hline OperOpiBita & 3.33 & 1 & 1.50 & 6.23 & 4.73 \\
\hline ProgMtoOpera & 3.20 & 1 & 1.45 & 5.64 & 4.19 \\
\hline
\end{tabular}


De lo anterior, claramente se observa que dos de las primeras cuatro actividades se relacionan con las cinco eses (HerraLugar y TjoLimOrde) y las otras dos con el compromiso de la gerencia (GerenEjem y DirPromParTrab). Asimismo, se observa que tres de esas actividades con valores de mediana más alta, también tienen la moda más alta, lo cual indica que la mayoría de los entrevistados concuerdan en que esas actividades son importantes para el éxito de los programas de TPM.

En orden ascendente y en relación con la mediana, también se observa que los valores más bajos son alcanzados por el involucramiento de la gerencia en proyectos de mantenimiento, la cooperación del operador en redacción de los reportes de bitácora (OperOpiBita) y programación de mantenimiento de máquinas y equipos con opinión de los operarios (ProgMtoOpera). Claramente se observa que esas actividades se relacionan con el involucramiento de terceras personas (operadores y gerencia) en asuntos de planeación y administración del mantenimiento, lo cual indica que no se desea tomar en cuenta a los operarios.

En relación con el rango intercuartílico, las actividades con menos variación se indican con asteriscos, las cuales se refieren a mantener áreas de trabajos limpios y ordenados (TjoLimOrde), a mantener la empresa limpia en todo momento y en todos los departamentos (EmpDtoLimp) y brindar entrenamiento para que los empleados realicen adecuadamente múltiples tareas (EntrMultTar). Esto confirma lo establecido anteriormente y se puede concluir que los encuestados tenían un mayor consenso en relación con éstas.

\section{Factibilidad del análisis factorial}

Se corrió una prueba de factibilidad para el análisis factorial y se encontró un índice KMO (Kaiser-Mayer-Olkin) de 0.898, el cual es considerado como aceptable. La prueba de esfericidad de Bartlett dio un valor de chi cuadrada aproximada de 1762.206 con 105 grados de libertad, lo que representa una significancia de 0.000. Además, con la finalidad de corroborar los resultados anteriores, se estimó el valor del determinante de la matriz de correlación, el cual fue de 0.000 . Con esto se concluye que el análisis factorial era factible y se procedió a aplicarlo.

\section{Análisis factorial}

En este análisis se identificaron tres factores con eigenvalor superior a la unidad y el gráfico de sedimentación que los representa se ilustra en la figura 3, donde a la 
altura del uno en el eje de las abscisas se ha trazado una línea paralela; así, en lo sucesivo, se analizan solamente los primeros tres componentes. En el cuadro 4 se ilustra el valor de cada uno de los eigenvalores, la varianza explicada por cada uno de ellos y la varianza acumulada. De esta forma se observa que con sólo tres componentes se logra explicar el $65.06 \%$ de la varianza total contenida en las quince actividades analizadas. Específicamente, con la rotación realizada, el primer componente explica el $29.85 \%$ de la variabilidad y está integrado por siete actividades; el segundo, el 20.78\%; y el tercero, el $14.43 \%$.

\section{Figura 3}

\section{Gráfica de sedimentación}

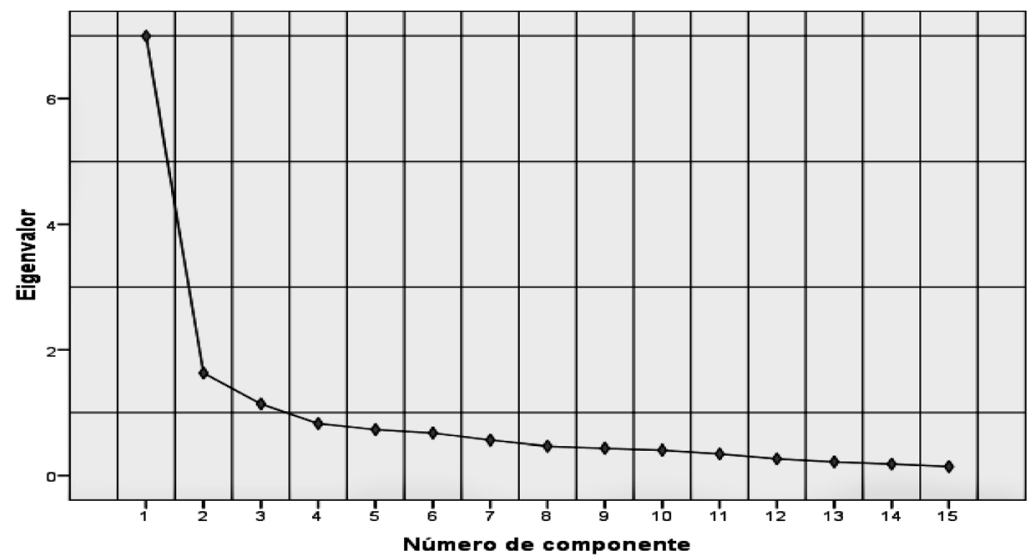

Cuadro 4

Varianza total explicada

\begin{tabular}{c|ccc|ccc}
\hline \multirow{2}{*}{$\begin{array}{c}\text { Compo- } \\
\text { nente }\end{array}$} & \multicolumn{3}{|c|}{ Eigenvalores iniciales } & \multicolumn{3}{c}{$\begin{array}{c}\text { Suma de las saturaciones al cuadrado } \\
\text { de la rotación }\end{array}$} \\
\cline { 2 - 7 } & Total & $\begin{array}{c}\text { \% de la } \\
\text { varianza }\end{array}$ & \% acumulado & Total & $\begin{array}{c}\text { \% de la } \\
\text { varianza }\end{array}$ & \% acumulado \\
\hline 1 & 6.99 & 46.63 & 46.63 & 4.48 & 29.85 & 29.85 \\
2 & 1.63 & 10.86 & 57.48 & 3.12 & 20.78 & 50.63 \\
3 & 1.14 & 7.57 & 65.06 & 2.16 & 14.43 & 65.06 \\
\hline
\end{tabular}


Con la información anterior, se procedió a identificar las actividades que conformaban cada uno de los componentes, para lo cual se hizo uso de la matriz de componentes rotados que se ilustra en el cuadro 5, en la que observa que siete actividades integran el primer componente, cuatro el segundo y, finalmente, tres al tercero. Se agrega, además, al lado derecho de este cuadro, en la tercera o última columna, una descripción del componente o factor.

Así, dadas las actividades que integran el primer componente se observa que éste está relacionado con el compromiso que tienen los directivos, gerencia y supervisión con el TPM; el segundo está asociado con la filosofía de las cinco eses; por último, el tercero está vinculado con la integración de los operadores en los programas de mantenimiento. Cabe mencionar que se comprueba nuevamente lo encontrado en el análisis unidimensional, donde la participación del operario era poco importante.

\section{Cuadro 5}

\section{Descripción de los componentes}

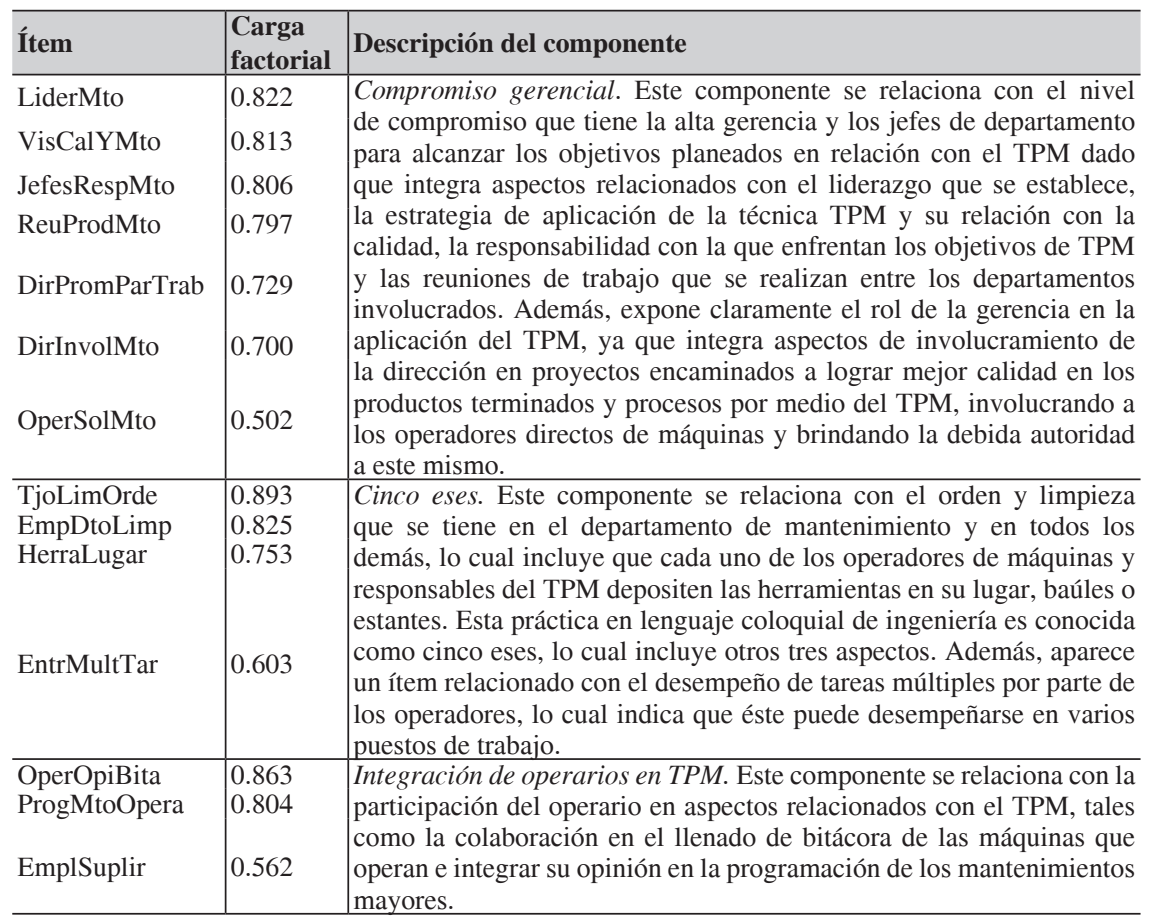




\section{Modelo de ecuaciones estructurales inicial}

Se generó un modelo inicial con base en los factores encontrados en el análisis factorial, el cual se ilustra en la figura 4 con los coeficientes de regresión estandarizados; dicho modelo está basado en la experiencia del autor, donde se supone, según el sentido de las líneas, que para obtener buenos resultados o beneficios de TPM, debe existir un amplio compromiso de la gerencia, las cinco eses e integración de los operarios en las actividades de TPM. De la misma manera, para que exista integración de los operarios se supone que debe haber cinco eses y compromiso de la gerencia y, finalmente, para que la disciplina de las cinco eses se dé, debe haber compromiso de la gerencia.

Figura 4

\section{Modelo inicial propuesto}

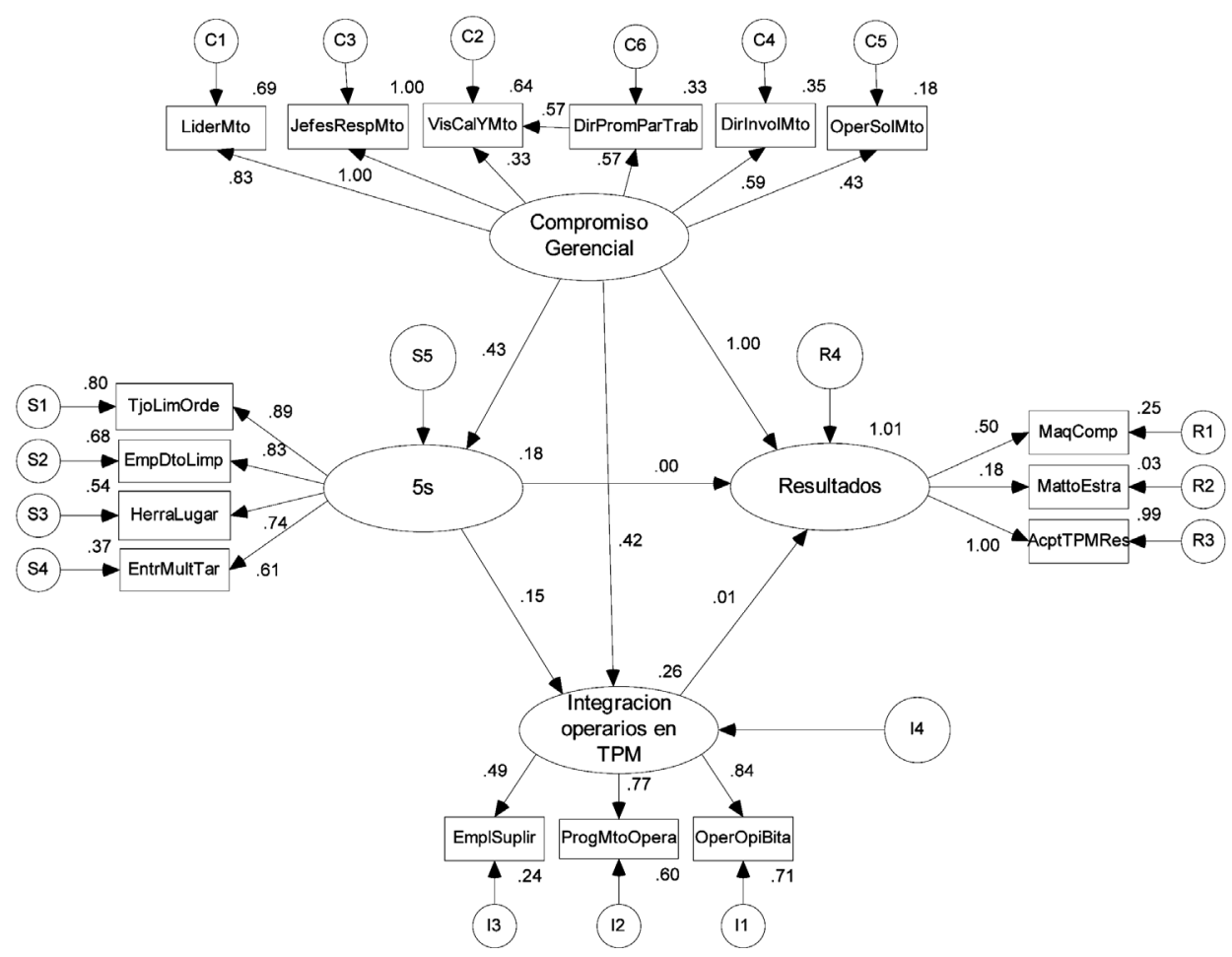

\section{Mejoramiento del modelo}

En el cuadro 6 se ilustran los modelos generados y las relaciones que fueron eliminándose y aquellas que fueron agregándose en cada uno de éstos, así como sus 
parámetros de eficiencia. Por ejemplo, el modelo inicial de la figura 4 tenía un valor de CMIN de 480.7 con 98 grados de libertad, lo que daba una relación CMIN/DF de 4.9 y un GFI de 0.711 , muy inferior al 0.9 requerido y el valor RMSEA fue de 0.139 , muy superior al 0.05 que se busca; sin embargo, se observó que el valor de la varianza del error C3 de la actividad JefesRespMto podía ser cero, por lo que se asignó ese valor al error, ganando así un grado de libertad y generando el modelo 2.

Los modelos posteriores se generaron de la misma manera; por ello, en la columna denominada Modificación del cuadro 6, se ha incluido una $A$ para indicar que la relación se ha agregado y una $E$ para indicar que la relación se ha eliminado. Claramente se observa que en los primeros seis modelos los grados de libertad se incrementan, dado que se agregan o quitan relaciones triviales, pero del siete en adelante, en cada modelo se pierde un grado de libertad.

Es importante señalar que de acuerdo con el índice CMIN/DF, desde el modelo seis se tiene un valor aceptable menor a cuatro; pero en relación con el CFI, el valor aceptable superior a 0.9 se obtiene hasta el modelo ocho, mientras que en relación con el GFI es aceptable hasta el modelo diez, lo mismo que para el RMSEA. Además, la máxima muestra que se requiere para hacer ese tipo de análisis fue de 90 elementos, lo cual fue menor a los 203 que se tenían.

\section{Cuadro 6}

\section{Índices de eficiencia de los MEE}

\begin{tabular}{|c|c|c|c|c|c|c|c|c|}
\hline Modelo & CMIN & DF & CMIN/DF & GFI & CFI & RMSEA & $\begin{array}{c}\text { HOELTER } \\
95\end{array}$ & Modificación \\
\hline Inicial & 480.7 & 98 & 4.90 & 0.711 & 0.863 & 0.139 & 52 & $\mathrm{C} 3=0$ \\
\hline 2 & 480.7 & 99 & 4.856 & 0.711 & 0.0863 & 0.138 & 52 & Cinco eses $\rightarrow$ Resultados \\
\hline 3 & 480.92 & & & & & & & Integración de Operarios en TPN \\
\hline 3 & 480.92 & 100 & 4.809 & 0.711 & 0.864 & 0.137 & 53 & $\rightarrow$ Resultados \\
\hline 4 & 482.59 & 101 & 4.778 & 0.711 & 0.863 & 0.137 & 53 & $\mathrm{R} 4=0$ \\
\hline 5 & 484.01 & 102 & 4.745 & 0.710 & 0.863 & 0.136 & 53 & $\begin{array}{c}\text { Cinco eses } \rightarrow \text { Integración d } \\
\text { Operarios en TPM }\end{array}$ \\
\hline 6 & 487.21 & 103 & 4.73 & 0.705 & 0.863 & 0.136 & 53 & $\mathrm{C} 2 \leftrightarrow 6$ \\
\hline 7 & 394.93 & 102 & 3.85 & 0.766 & 0.896 & 0.119 & 66 & EmplSuplir $\rightarrow$ EntMulTar \\
\hline 8 & 349.48 & 101 & 3.46 & 0.786 & 0.911 & 0.091 & 73 & DirPromParTrab $\rightarrow$ LiderMto \\
\hline 9 & 309.183 & 100 & 3.092 & 0.816 & 0.925 & 0.068 & 82 & DirPromParTrab $\rightarrow$ DirInvolTjo \\
\hline 10 & 278.12 & 99 & 2.809 & 0.908 & 0.936 & 0.046 & 90 & DirPromParTrab $\rightarrow$ MaqComp \\
\hline 11 & 246.28 & 98 & 2.513 & 0.918 & 0.947 & 0.038 & 101 & $\mathrm{C} 2 \leftrightarrow \mathrm{C} 1$ \\
\hline 12 & 234.19 & 97 & 2.414 & 0.929 & 0.951 & 0.031 & 110 & No se justicia el cambio en $\mathrm{CF}$ \\
\hline
\end{tabular}


Así, se generaron un total de doce modelos, pero en el último se observa que no se justifica agregar la relación de covarianza entre $\mathrm{C} 2$ y C1, dado que el incremento en el CFI es menor al 0.01 , por lo que el modelo final es el número diez y se ilustra en la figura 5 con los coeficientes de regresión estandarizados.

Figura 5

Modelo final

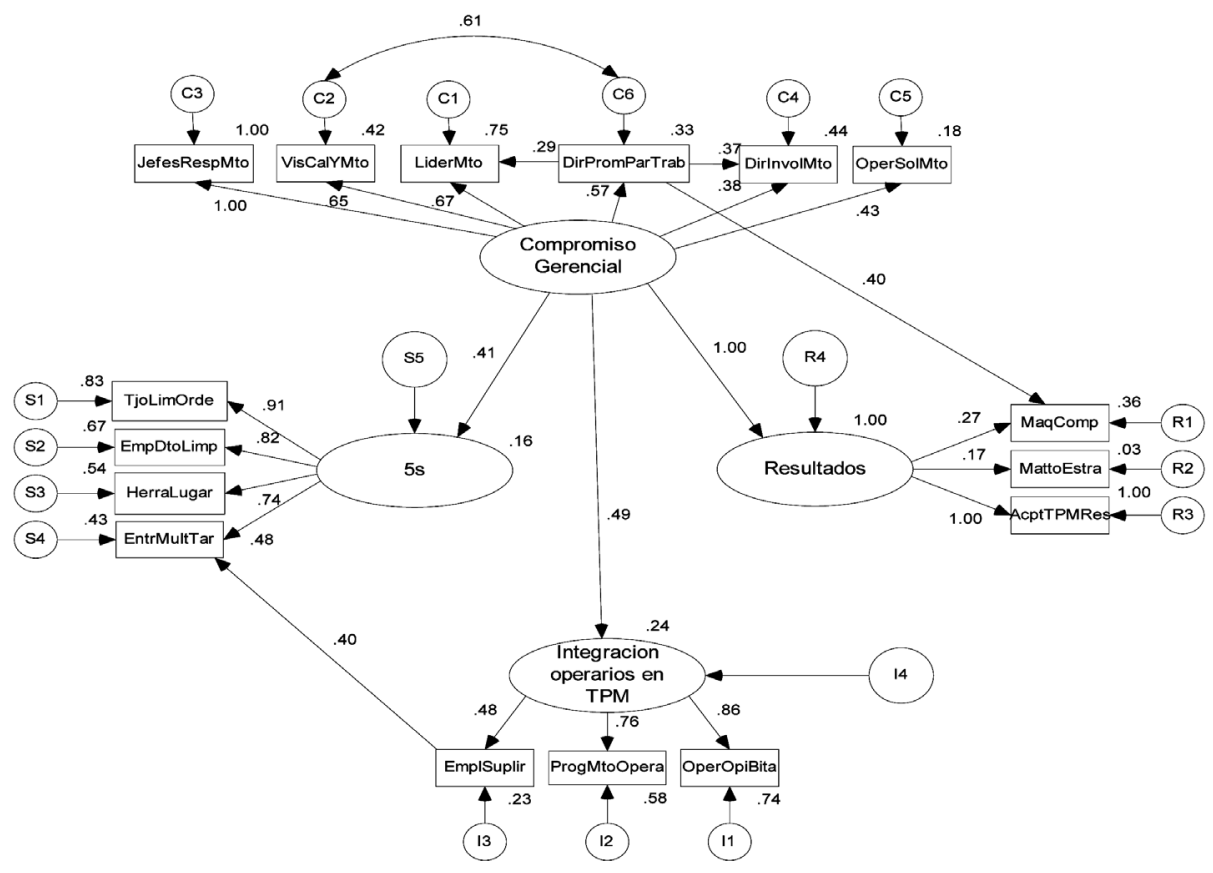

\section{Conclusiones y recomendaciones}

Con base en el modelo final se puede concluir que el único factor independiente es el compromiso gerencial, ya que es la única variable latente que no es explicada por otras, dado el sentido de las flechas que indican la relación con las demás variables. Si se logra tener un compromiso gerencial, se obtendrá:

- Una cultura de cinco eses, lo que equivale a decir que las herramientas y accesorios estén en su lugar, los sitios de trabajos sean limpios y ordenados, además de que los trabajadores podrán ser multifuncionales. El coeficiente 
de correlación múltiple es 0.16 y es significativo; adicionalmente, por cada unidad de varianza en compromiso gerencial, cinco eses, lo hace en 0.41 unidades, de acuerdo con los coeficientes estandarizados.

- Una integración de los operarios en las actividades de TPM, donde éstos puedan suplir a sus compañeros en diferentes lugares de trabajo y sean considerados en el registro de las bitácoras de trabajo y en la planeación de los paros programado por mantenimiento. El coeficiente de correlación múltiple es 0.24 y es significativo; además, por cada unidad de varianza en compromiso gerencial, integración operarios en TPM lo hace en 0.49 unidades, de acuerdo con los coeficientes estandarizados.

- Además, al lograr el compromiso gerencial, se obtendrán los tres beneficios buscados en TPM; es decir, la maquinaria y equipo de la empresa ayudan a obtener una ventaja competitiva en el sector en que se desarrolla la empresa, el TPM se convierte en una estrategia para la calidad y la programación de actividades por realizar en las líneas de producción y, por último, todos los directivos dentro de la empresa aceptan su responsabilidad hacia este programa. Cabe señalar que por cada unidad de varianza en compromiso gerencial, los resultados también lo hacen en una unidad, por lo que se concluye que existe una relación directa.

De la misma manera, en el modelo final se aprecian relaciones entre las variables observadas (en rectángulo), por ejemplo:

- Se requiere que los empleados sean capacitados en múltiples tareas (EntrMultTar) para poder suplir a sus compañeros en diferentes puestos (EmplSuplir) y por cada unidad de varianza que se registre en la segunda, la primera lo hará en 0.40 unidades.

- Se requiere que la alta gerencia promueva la participación de los trabajadores en las actividades de TPM (DirPromParTrab) para lograr que la maquinaria y equipo se conviertan en una ventaja competitiva para la empresa (MaqComp). Por cada unidad de varianza que ocurra en la primera, la segunda lo hace en 0.40 .

- Además, se requiere que la alta gerencia promueva la participación de los trabajadores en las actividades de TPM (DirPromParTrab) para lograr que el departamento de mantenimiento (LiderMto) asuma el liderazgo en dicho programa. Por cada unidad de varianza que ocurra en la primera, la segunda lo hace en 0.29 . 
Así, con base en el modelo final, se puede concluir que el compromiso de la gerencia es el más importante para garantizar los beneficios que ofrece el TPM y una de las actividades que debe esforzarse por realizar es promover la participación de los empleados.

\section{Referencias}

Aichlmayr, M. (2009). TPM: Healthcare for Equipment. Material Handling Management 64 (10): 18-20.

Aissani, N., B. Beldjilali y D. Trentesaux (2009). Dynamic scheduling of maintenance tasks in the petroleum industry: A reinforcement approach. Engineering Applications of Artificial Intelligence 22 (7): 1089-1103.

Alsyouf, I. (2009). Maintenance practices in Swedish industries: Survey results. International Journal of Production Economics 121 (1): 212-223.

Ashayeri, J. (2007). Development of computer-aided maintenance resources planning (CAMRP): A case of multiple CNC machining centers. Robotics and Computer Integrated Manufacturing 23 (6): 614-623.

Bentler, P.M. (1990). Comparative fit indexes in structural models. Psychological Bulletin 107: 238-246.

Bollen, K.A. y J. Liang (1988). Some properties of Hoelter's CN. Sociological Methods and Research 16: 492-503.

Browne, M.W. y R. Cudeck (1993). Alternative ways of assessing model fit. En K.A. Bollen y J.S. Long [Eds.]. Testing structural equation models. Newbury Park, CA: Sage: 136-162.

Byrne, B.M. (1989). A primer of LISREL: Basic applications and programming for confirmatory factor analytic models. New York: Springer-Verlag.

Carmines, E.G. y J.P. McIver (1981). Analyzing models with unobserved variables. En G.W. Bohrnstedt y E.F. Borgatta [Eds.]. Social measurement: $\mathrm{Cu}$ rrent issues. Beverly Hills: Sage. 
Chan, F.T.S., H.C.W. Lau, R.W. Ip, L.H.K. Chan y S. Kong (2005). Implementation of total productive maintenance: A case study. International Journal of Production Economics 95(1): 71-94.

Chand, G. y B. Shirvani (2000). Implementation of TPM in cellular manufacture. Journal of Materials Processing Technology 103 (1): 149-154.

Chandra, P. y T. Shastri (1998). Competitiveness of Indian manufacturing: findings of the 1997 manufacturing futures survey. Vikalpa 23(3): 25-36.

Cochran, W.G. (1952). The $\chi^{2}$ test of goodness of fit. Annals of Mathematical Statistics 23 (3): 315-345.

Cooke, F. L. (2000). Implementing TPM in plant maintenance: some organizational barriers. International Journal of Quality \& Reliability Management 17 (9): 1003-1016.

Cronbach, L. J. (1951). Coefficient alpha and the internal structure of tests. Psychometrika 16: 297-334.

Cua, K.O., K.E. McKone y R.G. Schroeder (2001). Relationships between implementation of TQM, JIT, and TPM and manufacturing performance. Journal of Operations Management 19 (6): 675-694.

Denneberg, D. y M. Grabisch (2004). Measure and integral with purely ordinal scales. Journal of Mathematical Psychology 48: 15-22.

Dinesh, S. y D. Tripathi (2006). Critical study of TQM and TPM approaches on business performance of Indian manufacturing industry. Total Quality Management \& Business Excellence 17 (7): 811-824.

Dowlatshahi, S. (2008). The role of industrial maintenance in the maquiladora industry: An empirical analysis. International Journal of Production Economics 114 (1): 298-307.

Eti, M.C., S.O.T. Ogaji y S.D. Probert (2004). Implementing total productive maintenance in Nigerian manufacturing industries. Applied Energy 79 (4): 385401. 
(2006). Reducing the cost of preventive maintenance (PM) through adopting a proactive reliability-focused culture. Applied Energy 83 (11): 1235-1248.

Gosavi, A. (2006). A risk-sensitive approach to total productive maintenance. Automatica 42 (8): 321-1330.

Gulliksen, H. y J. W. Tukey (1958). Reliability for the law of comparative judgment. Psychometrika 23: 95-110.

Hoelter, J. W. (1983). The analysis of covariance structures: Goodness-of-fit indices. Sociological Methods and Research 11: 325-344.

Jöreskog, K.G. (1969). A general approach to confirmatory maximum likelihood factor analysis. Psychometrika 34: 183-202.

y D. Sörbom, D. (1984). LISREL-VI user's guide. 3rd ed. Mooresville, IN: Scientific Software.

\section{Software.}

(1989). LISREL-7 user's reference guide. Mooresville, IN: Scientific

Lévy, J. P. y M. Varela (2003). Análisis multivariable para las ciencias sociales. Madrid: Prentice Hall.

Likert, R. (1932). A Technique for the measumerement of attitudes. Archives of Psychology 22 (140): 1-55.

Marsh, H.W. y D. Hocevar (1985). Application of confirmatory factor analysis to the study of self-concept: First- and higher-order factor models and their invariance across groups. Psychological Bulletin 97: 562-582.

McDonald, R. P. y H. W. Marsh (1990). Choosing a multivariate model: Noncentrality and goodness of fit. Psychological Bulletin 107 (2): 247-255.

McKone, K. E., R.G. Schroeder y K.O. Cua (1999). Total productive maintenance: a contextual view. Journal of Operations Management 17(2): 123-144. 
(2001). The impact of total productive maintenance practices on manufacturing performance. Journal of Operations Management 19 (1): 39-58.

Nunally, J.C. (1978). Psychometric theory, New York: McGraw-Hill.

y H. Bernstein (2005). Teoría psicométrica, México: McGraw-Hill Interamericana.

Pinjala, S.K., L. Pintelon y A. Vereecke (2006). An empirical investigation on the relationship between business and maintenance strategies. International Journal of Production Economics, 104 (1), 214-229.

Pollandt, S. y R. Wille (2005). Functorial scaling of ordinal data. Discrete Applied Mathematics 147 (1): 101-111.

Reed, R. (1996). Beyond process: TQM content and firm performance. Academy of Management Review 21 (1): 173-202.

Sachdeva, A., D. Kumar, D. y P. Kumar (2008). Planning and optimizing the maintenance of paper production systems in a paper plant. Computers \& Industrial Engineering 55 (4): 817-829.

Steiger, J. H. y J. C. Lind (1985). Statistically-based tests for the number of common factors. Paper presented at the Annual Spring Meeting of the Psychometric Society, Iowa City.

Streiner, D. y G. R. Norman (1995). Health Measurement Scales. A Practical Guide to their Development and Use, 2a. ed. Oxford: Oxford University Press.

Tajiri, M. y F. Gotoh (1992). TPM Implementation: A Japanese Approach. New York: McGraw Hill.

Takahashi, Y. y T. Osada (1989). TPM: Total Productive Maintenance. MA: Productivity Press.

Tanaka, J. S. y G.J. Huba (1985). A fit index for covariance structure models under arbitrary GLS estimation. British Journal of Mathematical and Statistical Psychology 38: 197-201. 
Tastle, W. J. y M. J. Wierman (2007). Using Consensus to Measure Weighted Targeted Agreement. Fuzzy Information Processing Society 24: 31-35.

Vinodh, S. (2010). Improvement of agility and sustainability: A case study in an Indian rotary switches manufacturing organization. Journal of Cleaner Production 18 (10): 1015-1020.

Wheaton, B., B. Muthén, D. F. Alwin y G. F. Summers (1977). Assessing reliability and stability in panel models. En D.R. Heise [Ed.]. Sociological methodology 1977. San Francisco: Jossey-Bass: 84-136.

Wikoff, D. (2007). Improve all the M's in TPM system. Plant Engineering 61 (12): 21-22.

Zhou, W. H. y G. L. Zhu (2008). Economic design of integrated model of control chart and maintenance management. Mathematical and Computer Modeling 47 (11): 1389-1395.

(A) 